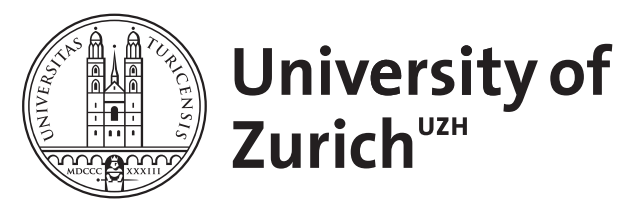

Zurich Open Repository and Archive

University of Zurich

University Library

Strickhofstrasse 39

CH-8057 Zurich

www.zora.uzh.ch

Year: 2013

\title{
Diagnose von tiefer Venenthrombose und Lungenembolie
}

Neuner-Jehle, S

Posted at the Zurich Open Repository and Archive, University of Zurich ZORA URL: https://doi.org/10.5167/uzh-93668

Journal Article

Published Version

Originally published at:

Neuner-Jehle, S (2013). Diagnose von tiefer Venenthrombose und Lungenembolie. PrimaryCare, 13(16):294295. 


\title{
Diagnose von tiefer Venenthrombose und Lungenembolie
}

\author{
Ein Beitrag aus der Reihe «Die Werkzeugkiste des Familienarztes»
}

Wie schon in einer früheren Folge beschrieben, sind akute Thoraxschmerzen ein häufiger Konsultationsgrund in der Hausarztpraxis. Ein akutes Koronarsyndrom oder eine Lungenembolie sind hingegen selten (in ca. 2\%) der Grund dafür [1], ganz im Gegensatz zu der Häufigkeit dieser bedrohlichen Ursachen im Setting der Spitalnotfallstation.

Damit wir unsere Point-of-Care-Diagnostik wie z.B. das D-Dimer zielführend einsetzen können, benötigen wir eine möglichst hohe Vortestwahrscheinlichkeit für das Risiko einer Lungenembolie. Wir sind dabei nicht nur auf die Intuition angewiesen: Es gibt validierte Kriterien, die uns eine Einschätzung ermöglichen, ob der Patient mit hoher Wahrscheinlichkeit an einer Lungenembolie leidet.

\section{Tiefe Venenthrombose}

Klassiker ist der Score von Wells aus dem Jahr 2003 [2] (Tab. 1). In Kombination mit einem D-Dimer erlaubt er eine recht gute Auslese, welche Patienten in die erweiterte (sonographische) Diagnostik gehören.

\section{Tabelle 1}

Wells-Score für tiefe Venenthrombose. Auswertung: 0 Punkte = geringe, $1-2$ Punkte $=$ mittlere, 3 Punkte oder mehr $=$ hohe Wahrscheinlichkeit für TVT

\begin{tabular}{ll} 
Kriterium & Punkte \\
\hline Umfangdifferenz der Unterschenkel $(3 \mathrm{~cm})$ & 1 \\
\hline Aktive Tumorerkrankung & 1 \\
\hline Einseitiges Ödem des Unterschenkels & 1 \\
\hline Beinschwellung (ganzes Bein) & 1 \\
\hline Schmerz am Unterschenkel (entlang der Venen) & 1 \\
\hline Erweiterte Kollateralvene & 1 \\
\hline Vorbestehende Immobilisation oder Parese & 1 \\
\hline Bettruhe länger als 3 Tage oder Operation innert & \\
der letzten 12 Wochen & 1 \\
\hline Frühere tiefe Venenthrombose & 1 \\
\hline Andere Diagnosen ebenso wahrscheinlich wie TVT & -2
\end{tabular}

\section{Tabelle 2}

Wells-Score für Lungenembolie. Auswertung: $<2$ Punkte = geringe, 2-6 Punkte $=$ mittlere, $>6$ Punkte $=$ hohe Wahrscheinlichkeit für LE

\section{Kriterium}

Punkte

Klinische Zeichen einer TVT (Beinschwellung,

strangförmiger Schmerz)

Diagnose LE wahrscheinlicher als jede andere Diagnose

$H f \geq 100 / m$

Bettruhe länger als 3 Tage oder Operation innert

der letzten 12 Wochen

Frühere tiefe Venenthrombose oder LE

1,5

Hämoptyse

Aktive Tumorerkrankung

\section{Lungenembolie}

Der Traditionelle: Wells-Score

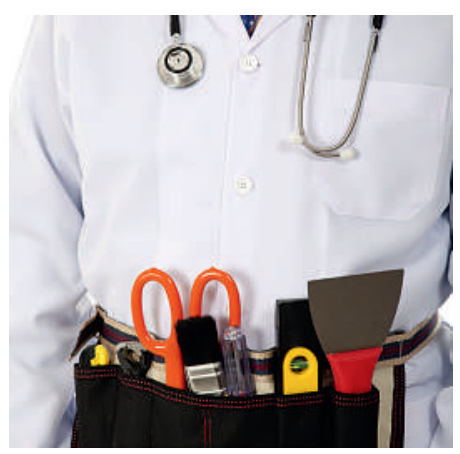

Seit über einem Jahrzehnt leistet der Score von Wells gute Dienste [3] (Tab. 2). Es existiert eine vereinfachte Variante, die mit denselben sechs objektiven plus einem subjektiven Kriterium arbeitet - nur die Punktevergabe variiert.

Der Moderne aus der Schweiz: Geneva-Score

Eine Genfer Forschungsgruppe legt in bereits revidierter Fassung einen Score vor, der aus acht objektiven Variablen besteht [4] (Tab. 3). Analog zum Wells-Score gibt es eine vereinfachte Version mit identischen Kriterien, aber anderer Punkteverteilung.

\section{Tabelle 3}

Revidierter Geneva-Score, vereinfachte Version, für Lungenembolie. Auswertung: $<2$ Punkte $=$ LE unwahrscheinlich; $>2$ Punkte $=$ LE wahrscheinlich .

\begin{tabular}{ll}
\hline Kriterium & Punkte \\
\hline Vorangegangene TVT oder Lungenembolie & 1 \\
\hline Herzfrequenz & \\
\hline $75-94 / m$ & 1 \\
\hline$>95 / m$ & 2 \\
\hline Operation oder Fraktur innert des letzten Monats & 1 \\
\hline Hämoptyse & 1 \\
\hline Aktives Melanom & 1 \\
\hline Einseitiger Beinschmerz & 1 \\
\hline Schmerz bei Wadenpalpation und einseitiges Ödem & 1 \\
\hline Alter über 65 Jahre & 1
\end{tabular}

Eine holländische Arbeit hat die Original- und die Kurzversion beider Scores (Wells/Geneva) in einer prospektiven Kohortenstudie miteinander verglichen: praktisch identische Wertigkeit aller Scores [5].

\section{Ein Wort zum D-Dimer: Schlechter als sein Ruf?}

Obwohl das D-Dimer über eine exzellente Ausschlusskraft verfügt, erlaubt ein negativer Wert beim Vorliegen von erheblichen klinischen Verdachtsmomenten (hoher Punktzahl bei den Scores) den Ausschluss einer Lungenembolie nicht. Mit anderen Worten: Bei hoher anamnestisch-klinischer Wahrscheinlichkeit für eine Lungenembolie dürfen wir einem negativen D-Dimer nicht trauen. Die klinische Risikostratifizierung ist ebenso wichtig, wenn nicht wichtiger, wie der Labortest!

Bei positivem Test ist die Lungenembolie wegen der falsch-positiven Resultate noch längst nicht gesichert (Tab. 4); auch dieser ist also mit Vorsicht zu geniessen und soll nicht automatisch zur Weiterabklärung oder Hospitalisation führen. 


\section{Tabelle 4}

Gründe für ein erhöhtes D-Dimer in Abwesenheit einer TVT oder LE.

Alter $>70$ (erhöhter Fibrinogenumsatz)

Entzündungsprozess

\section{Malignome}

Leberinsuffizienz

Schwangerschaft

Trauma oder OP letzte 4 Wochen

Gerinnungshemmende Therapie

Aortenaneurysma/-dissektion, akuter arterieller Verschluss

\section{Fazit}

Bei beiden Lungenembolie-Scores erstaunt mich die Hämoptyse: Im klinischen Alltag habe ich sie kaum je bei einer Lungenembolie gesehen. Dafür fehlt der Thoraxschmerz in den Scores, dem ich oft bei Lungenembolie begegne. Voilà die Limiten der kleinen Fallzahlen, die wir als Einzelne überblicken!

Die Stärke der Kombination «Score plus D-Dimer» liegt in der Ausschlusskraft - «nur» etwa 1,5\% der tiefen Venenthrombosen und 0,5\% der Lungenembolien werden verpasst, wenn der Score niedrig ist, das D-Dimer negativ ausfällt und dementsprechend auf eine Weiterabklärung verzichtet wird. Welcher der vorgestellten Scores verwendet wird, ist dabei nicht Match-entscheidend.

\section{Literatur}

1 Cayley WE Jr. Diagnosing the cause of chest pain. Am Fam Physician. 2005;72(10):2012-21.

2 Wells PS, Anderson DR, Rodger M, Forgie M, Kearon C, et al. Evaluation of D-dimer in the diagnosis of suspected deep-vein thrombosis. N Engl J Med. 2003;349(13):1227-35.

3 Wells PS, Anderson DR, Rodger M, Stiell I, Dreyer JF, et al. Excluding pulmonary embolism at the bedside without diagnostic imaging: management of patients with suspected pulmonary embolism presenting to the emergency department by using a simple clinical model and d-dimer. Ann Intern Med. 2001;135(2):98-107.

4 Le Gal G, Righini M, Roy PM, Sanchez O, Aujesky D, et al. Prediction of pulmonary embolism in the emergency department: the revised Geneva score. Ann Intern Med. 2006;144(3):165-71.

5 Douma RA, Mos IC, Erkens PM, Nizet TA, Durian MF, et al. Performance of 4 clinical decision rules in the diagnostic management of acute pulmonary embolism: a prospective cohort study. Ann Intern Med. 2011;154(11):709-18.

Korrespondenz:

Dr. med. Stefan Neuner-Jehle, MPH

Institut für Hausarztmedizin Zürich

Pestalozzistrasse 24

8091 Zürich

sneuner[at]bluewin.ch 